\title{
Study on Dynamic Model of Metallic Jet Penetration into Soil Based on Compression Theories
}

\author{
Yu-ling Zhang ${ }^{*}$, Guo-qing Liu, Xing-yong Gao, Jian-wei Zhen \\ Ordnance Engineering College, 050003Shijiazhuang, China \\ * zhangyuling2009@163.com
}

Key Words: Dynamic Model, Metallic Jet, Soil, Compression Theories

Abstract: Based on classic theories of soil compression and metallic jet penetration, this paper built a dynamic model of metallic jet penetration into soil, and verified its rationality through tests. The paper applied this model to the theoretical analysis of the process of metallic jet penetration into soil, and obtained variation laws of soil density, jet tip velocity, penetration velocity and stagnation pressure along with penetration depth.

\section{Introduction}

Unexploded ordnance (UXO) may occur whether during range tests or in military target practice exercises, falling to ground surface, or penetrating into soil. As for the unexploded ordnance, leaderships of the $\mathrm{CMC}$ and headquarters have demanded that "overhauls should be carried out on unexploded ordnance, which should be destroyed completely on the spot in a timely manner, and prohibited from removal at will". Therefore, how to destroy the unexploded ordnance penetrated into soil has become an urgent issue to be addressed. One of the solutions for this problem is using Munroe effect to generate metallic jet to destroy the buried unexploded ordnance, and extensive researches on this have been done by scholars both in China and around the world ${ }^{[1-3]}$. However, none of the existing researches has fully considered the factor of soil dynamic performance under metallic jet, moreover, due to the compressibility of soil, the process and features of jet penetration into soil obviously differ from those of jet penetration into metal plate and cement, and the existing theoretical models of using metallic jet to study various incompressible objects will not apply directly to the analysis of metallic jet penetration into soil. Hence, based on classic theories of soil compression and metallic jet penetration, studied the dynamic model of metallic jet penetration into soil.

\section{Theoretical model of metallic jet penetration into soil}

\subsection{Problem Analysis and Assumption.}

Soil will be compressed under external pressure, and similarly, the compressive phenomenon also presents when metallic jet penetrates into soil. Study shows that, when super high-speed jet penetrates into a plate, the interior of the plate will form a stress wave, as successive jet continues to provide energy supply, it will form a shock wave impacted area at some distance from the penetration interface, so the metallic jet penetration into soil is a complex and comprehensive process where both compression and penetration have been present. As indicated in Fig. 1(a), evenly divide soil with thickness of $x$ into $\mathrm{n}$ layers, with thickness of $x / \mathrm{n}$ for each layer. The thickness of the 1st layer is represented with $x_{1}$, that of the 2nd layer with $x_{2}$, that of the layer with $x_{\mathrm{i}}$, that of the nth layer with $x_{\mathrm{n}}$. Then $x_{1}=x_{\mathrm{i}}=x_{\mathrm{n}}=x / n$. 


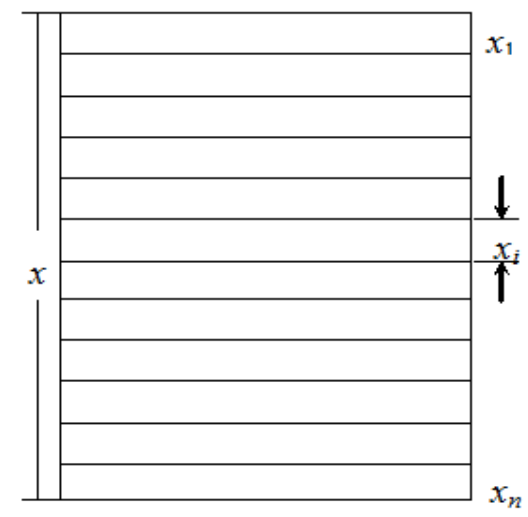

(a)

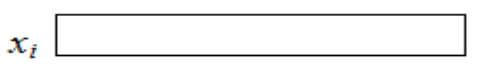

(b)

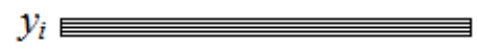

(c)

Figure 1. Hierarchical processing of soil

Hence, the process of metallic jet compression and penetration into soil with thickness of $x$ is the process of successive compression and penetration into the 1st, 2 nd, to nth layers of soil. As indicated in Fig. 1 (b), as for soil with thickness of $x$, when $\mathrm{n}$ is big enough, and the thickness of the ith layer $x_{\mathrm{i}}$ is small enough, the process of jet compression and penetration into the ith layer of soil may be viewed as the equivalent of two stages: stage one is the stage of jet compressing soil, where we suppose that jet only compresses soil but does not penetrate surface of the compressed soil, until the compressed soil could be regarded as incompressible material with constant density, as indicated in Fig.1(c), when the thickness of the soil is $y_{i}$; stage two is the stage of jet penetration into the compressed soil, where jet only penetrates soil but does not compress it, with soil density unchanged, until completion of penetration into the incompressible soil with thickness of $y_{\mathrm{i}}$, the compression and penetration into the (i+1)th layer of soil begin. The process of jet penetration into the (i+1)th layer of soil is similar to that of penetration into the ith layer of soil, until the completion of compression and penetration into the nth layer of soil.

\subsection{Construction of Compression Model.}

There are many soil compression models, and among them, the soil compression model proposed by Assouline and others has better feasibility whether in the sense of physics or scope of application, and the expression of Assouline model for the ith layer of soil is written ${ }^{[4]}$ :

$$
\frac{\rho_{s i}-\rho_{s 0}}{\rho_{s \max }-\rho_{s 0}}=\left[1-e^{-\left(\kappa \sigma_{i}\right)^{\omega}}\right]
$$

Where $\rho_{s i}$ is density of the ith layer of soil during compression process, $\mathrm{kg} / \mathrm{m}^{3} ; \rho_{s \max }$ is the maximum density of the soil possible to be compressed, $\mathrm{kg} / \mathrm{m}^{3} ; \rho_{s 0}$ is the soil density before metallic jet compression, $\mathrm{kg} / \mathrm{m}^{3} ; \kappa$ is simulation parameter; $\omega$ is simulation parameter; $\sigma_{i}$ is pressure, Pa. Transforming Eq.1, we obtain:

$$
\rho_{s i}=\rho_{s 0}+\left(\rho_{s \max }-\rho_{s 0}\right)\left[1-e^{-\left(\kappa^{\omega}\right)\left(\sigma_{i}^{\omega}\right)}\right]
$$

Let $A=\rho_{\text {smax }}-\rho_{s 0} ; B=\kappa^{\omega} ; C=\omega$; Obviously, $A>0$, then Eq. 2 is transformed into: 


$$
\rho_{s i}=\rho_{s 0}+A\left[1-e^{-B\left(\sigma_{i}^{c}\right)}\right]
$$

We take Eq.3 as the soil compression model for research, $A, B$, and $C$ are simulation parameters.

\subsection{Construction of Penetration Model.}

As for the ith layer of the compressed soil, in the process of metallic jet penetration into soil, the pressure at the interface of metallic jet and the soil is written:

$$
\sigma_{i}=\frac{1}{2} \rho_{s i} U_{i}^{2}
$$

Where $\rho_{s i}$ soil density in penetration is process, and $U_{i}$ is velocity of jet penetration into soil. From Bernoulli equation, we get:

$$
\frac{1}{2} \rho_{j}\left(V_{j i}-U_{i}\right)^{2}=\frac{1}{2} \rho_{s i} U_{i}^{2}
$$

Combining Eq.3, Eq.4, and Eq.5, we obtain:

$$
V_{j i}=1.414\left(\frac{1}{\sqrt{\rho_{s i}}}+\frac{1}{\sqrt{\rho_{j}}}\right)\left[\frac{-B}{\ln \left(1-\frac{\rho_{s i}-\rho_{s 0}}{A}\right)}\right]^{2 C}
$$

This is the relationship of metallic jet velocity and soil density during metallic jet penetration into soil.

When the ith layer of soil is compressed and starts to be penetrated, the soil layer could be regarded as quasi-steady ideal incompressible fluid, then the relationship of penetration depth and jet velocity is written ${ }^{[5]}$ :

$$
y_{i}=(H-b)\left[\left(\frac{V_{j(i-1)}}{V_{j i}}\right)^{\sqrt{\frac{\rho_{j}}{\rho_{s i}}}}-1\right]
$$

Where $V_{j i}$ is the jet tip velocity after penetrating the ith layer of soil; $V_{j(i-1)}$ is jet tip velocity before penetrating the ith layer of soil; $y_{i}$ is the thickness of the ith layer of soil after being compressed; $H$ is standoff; $b$ is the distance from virtual origin to liner mouth (about half height of liner); $\rho_{s i}$ is soil density; $\rho_{j}$ is liner density.

We suppose that soil is only compressed in an axial direction of penetration, and take the soil element of some thickness to study, according to law of conservation of mass, before and after the soil is being compressed, there are:

$$
x_{i} \rho_{s 0}=y_{i} \rho_{s i}
$$

Where $x_{i}$ and $y_{i}$ are thickness of the soil element before and after being compressed, respectively; $\rho_{s 0}$ and $\rho_{s i}$ are densities of the soil element before and after being compressed, respectively $=1,2$, , n.

As for penetration into the ith layer of soil, substituting the Eq.7 into Eq.8, we obtain: 


$$
x_{i}=\frac{\rho_{s i}}{\rho_{s 0}}(H-b)\left[\left(\frac{V_{j(i-1)}}{V_{j}}\right)^{\sqrt{\frac{\rho_{j}}{\rho_{s i}}}}-1\right]
$$

As for computing compression and penetration into the ith layer of soil, we combine Eq.4, Eq.5, and Eq.6, and obtain pressure expression in the penetration process, and combine Eq.6 and Eq.9, an equation group could be given:

$$
\left\{\begin{array}{l}
\sigma_{i}=\left[-\frac{B}{\ln \left(1-\frac{\rho_{s i}-\rho_{s 0}}{A}\right)}\right]^{C} \\
x_{i}=\frac{\rho_{s i}}{\rho_{s 0}}\left((H-b)\left[\left(\frac{V_{j(i-1)}}{V_{j i}}\right)^{\sqrt{\rho_{j} / \rho_{s i}}}-1\right]\right) \\
V_{j i}=1.414\left(\frac{1}{\sqrt{\rho_{s i}}}+\frac{1}{\sqrt{\rho_{j}}}\right)\left[\frac{-B}{\ln \left(1-\frac{\rho_{s i}-\rho_{s 0}}{A}\right)}\right]^{2 C}
\end{array}\right.
$$

Eq.10 is theoretical model of metallic jet compression and penetration into soil, where $x_{i}$ is the thickness of the ith layer of soil; $V_{j(i-1)}$ is jet tip velocity before penetration into soil with thickness of $x_{i} ; V_{j i}$ is jet tip velocity after penetration into soil with thickness of $x_{i}$. When metallic jet penetrates into the (i+1)th layer of soil, in Eq.10 $V_{j(i-1)}$ changes to $V_{j i}$, and $V_{j i}, x_{i}, U_{i}, \rho_{s i}$ change to $V_{j(i+1)}, x_{(i+1)}, U_{(i+1)}$ and $\rho_{s(i+1)}$, respectively.

\section{Metallic jet penetration test}

We select shaped charge structure with caliber of $50 \mathrm{~mm}$, and its detonation forms metallic jet, which travels a distance of preferred standoff by air motion and penetrates into soil medium of certain thickness, and we adopt pulsed x-ray machine to test jet tip velocity and diameter after penetration out of soil medium, and the experimental arrangement is set as indicated in Fig. 2. Supported by supporting cylinder cut out of PVC tube, the shaped charge is placed on soil medium, whose height depends on preferred standoff of the shaped charge. Soil is filled into the PVC tube with bore of $15 \mathrm{~cm}$. According to the position of X-ray probe in bomb tower, we make the lower edge of soil medium $150 \mathrm{~cm}$ high from ground surface, support the whole explosive system with a framework, record image position variations of X-ray on the negative film at different times to deduce and compute metallic jet velocity, and to calculate metallic jet diameter by comparing metallic jet diameter in the images with the scale.

We select steel balls with diameter of $19.82 \mathrm{~mm}$ as scale, and place them at the vertical plane, parallel with the negative film, where metal jet will travel, and the steel balls and metallic jet will have the same coefficient of amplification on the negative film. Assume that the diameter of steel ball on the negative film is $d$, then the coefficient of amplification of this test is $\lambda, \lambda=d / 19.82$.

If the length of metallic jet after penetrating soil is $l^{\prime}$, it's true length is: $l=l^{\prime} / \lambda$.

Note that if at moment $t_{1}$, the length of metallic jet on the negative film after penetrating into soil is $l_{1}^{\prime}$, after some time, at moment $t_{2}$, the length of metallic jet on the negative film after penetrating into soil is $l_{2}^{\prime}$, then the average velocity of metallic jet in the time interval from $t_{1}$ to $t_{2}$ is: 


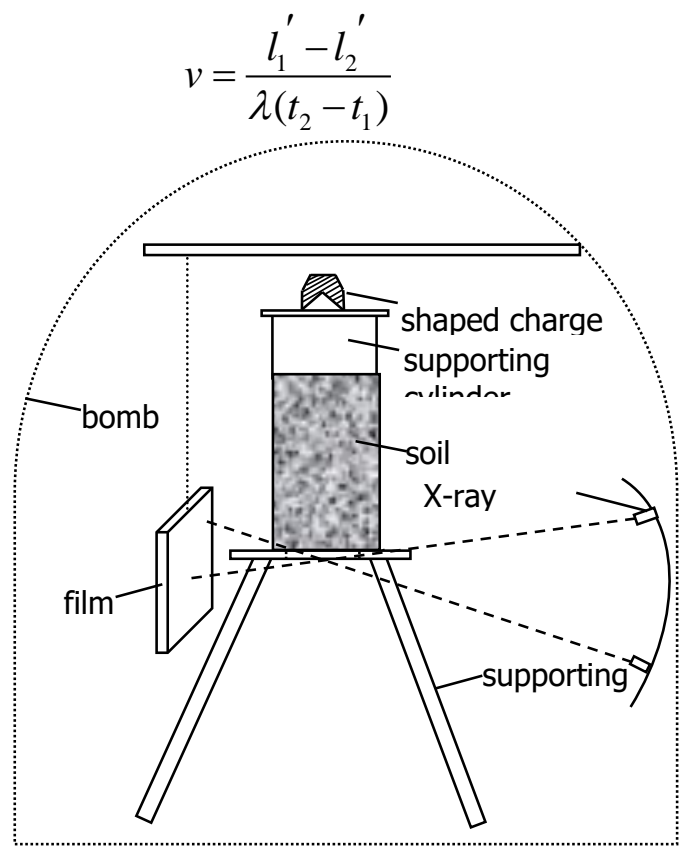

Figure 2. Principle of test

As jet tip travels very fast, usually above $7000 \mathrm{~m} / \mathrm{s}$, at a very short distance, this average velocity could be used to represent jet velocity after penetrating soil.

The test adopts shaped charge JO-8, with detonation velocity $8700 \mathrm{~m} / \mathrm{s}$, density $1.840 \mathrm{~g} / \mathrm{cm}^{3}$, and red copper liner with density of $8.9 \mathrm{~g} / \mathrm{cm}^{3}$.

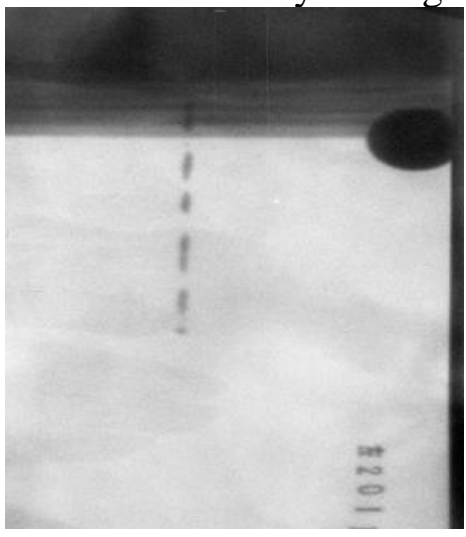

(a) $105 \mu \mathrm{s}$

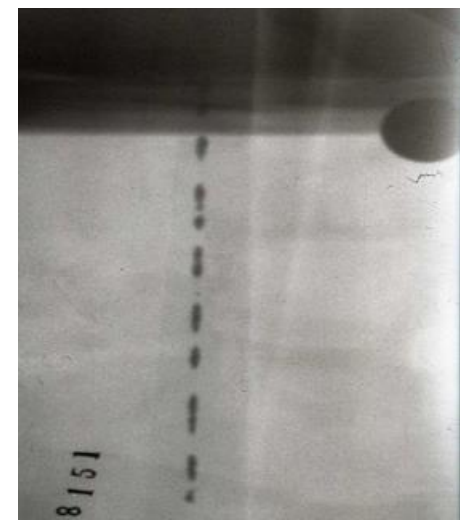

(b) $118 \mu \mathrm{s}$

Figure 3. X-ray images of metallic jet penetration into soil at different times

Fig. 3 is X-ray photographs of metallic jet penetration into experimental soil at different times, and we determine coefficient of amplification of each picture by measuring steel ball scale, and combining with specific moment of each picture taken and motion displacement of jet tip, we may obtain metallic jet tip velocity and tip diameter after penetration out of sand at the preferred standoff of the munitions. After several tests, we obtain the jet tip velocities of penetrating soil with thickness of $20 \mathrm{~cm}, 30 \mathrm{~cm}, 40 \mathrm{~cm}, 50 \mathrm{~cm}, 60 \mathrm{~cm}, 70 \mathrm{~cm}$ and $80 \mathrm{~cm}$, respectively, see Table 1.

Table 1. Calculation table for verification of theoretical model

\begin{tabular}{|c|c|c|c|c|}
\hline \multirow{2}{*}{ Serial No } & \multirow{2}{*}{$\begin{array}{l}i x / \\
\mathrm{cm}\end{array}$} & test results & $V_{i ;} /(\mathrm{m} / \mathrm{s})$ & calculation results \\
\cline { 3 - 5 } & 20 & 4795 & - & relative error(\%) \\
\hline 1 & 30 & 4006 & 4078 & 1.8 \\
\hline 2 & 40 & 3615 & - & - \\
\hline 3 & 50 & 3217 & 3339.37 & 3.8 \\
\hline 4 & 60 & 3155 & - & 2.2 \\
\hline 5 & 70 & 2956 & 3020.148 & 4.3 \\
\hline 6 & 80 & 2785 & 2903.99 & \\
\hline 7 & & & & \\
\hline
\end{tabular}




\section{Application of theoretical model}

\subsection{Verification of Theoretical Model.}

From equation group(10),we learn that in order to obtain jet tip velocity, penetration velocity and depth in the penetration process, we must first calculate and determine the values of coefficients A, $\mathrm{B}, \mathrm{C}$, and then solve the equation group. While calculating and determining the values of coefficients $\mathrm{A}, \mathrm{B}, \mathrm{C}$ of the equation group requires at least impact velocities $V_{j(i-1)}$ and outgoing velocities $V_{j i}$ of three sets of metallic jets penetration into soil element, by solving Eq. 10 to obtain its value. We take from Table 1 . data of penetration into soil with thickness of $20 \mathrm{~cm}, 40 \mathrm{~cm}$ and $60 \mathrm{~cm}$, obtain after calculating the values parameters $A, B, C$ are: $A=2109.5 \mathrm{~kg} / \mathrm{m}^{3}, B=2.12 \times 10^{-25}, C=2.51$, respectively. Substituting values of parameters $A, B, C$ into Eq.10, we may obtain:

$$
\left\{\begin{array}{l}
x_{i}=\frac{\rho_{s i}}{\rho_{s 0}}(H-b)\left[\left(\frac{V_{j(i-1)}}{V_{j i}}\right)^{\sqrt{\frac{\rho_{j}}{\rho_{s i}}}}-1\right] \\
V_{j i}=1.16 \times 10^{5}\left(\frac{1}{\sqrt{\rho_{s i}}}+\frac{1}{\sqrt{\rho_{j}}}\right)\left[-\ln \left(1-\frac{\left.\rho_{s i}-\rho_{s 0}\right)}{2109.5}\right)\right]^{0.199} \\
U_{i}=\frac{1.16 \times 10^{5}}{\sqrt{\rho_{s i}}}\left[-\ln \left(1-\frac{\rho_{s i}-\rho_{s 0}}{2109.5}\right)\right]^{0.199}
\end{array}\right.
$$

Eq.12 is the model of metallic jet compression and penetration into the experimental soil, from Eq.12, we learn that, adoption of iterative method may obtain the relation of penetration depth $\sum_{n=1}^{n=i} x_{i}$, jet tip velocity $V_{j i}$, penetration velocity $U_{i}$ with soil density $\rho_{s i}$, and thus jet tip velocity, penetration velocity and soil density of any penetration depth could be obtained through calculation. The obtained penetration depths are jet tip velocity at $0.3 \mathrm{~m}, 0.5 \mathrm{~m}, 0.7 \mathrm{~m}$ and $0.8 \mathrm{~m}$, See Table 1 ., where we learn that, the relative error between theoretical calculation results and experimental results is small, which indicates that the theory model constructed is feasible.

\subsection{Application of Theoretical Model.}

Fig. 4, Fig. 5, Fig. 6 and Fig. 7 are charts indicating variation law of soil density, metallic jet tip velocity, penetration velocity and stagnation pressure along with penetration depth respectively, which show that soil density does not change much at first along with penetration depth, then decreases considerably within some extent of penetration depth, at last, the declining tendency becomes gentle; jet tip velocity, penetration velocity and stagnation pressure along with penetration depth decrease dramatically at first, then followed by a trend of flat lining, where the dramatic declining process of stagnation pressure is even more remarkable, due to its relationship of quadratic product of soil density and penetration velocity. 


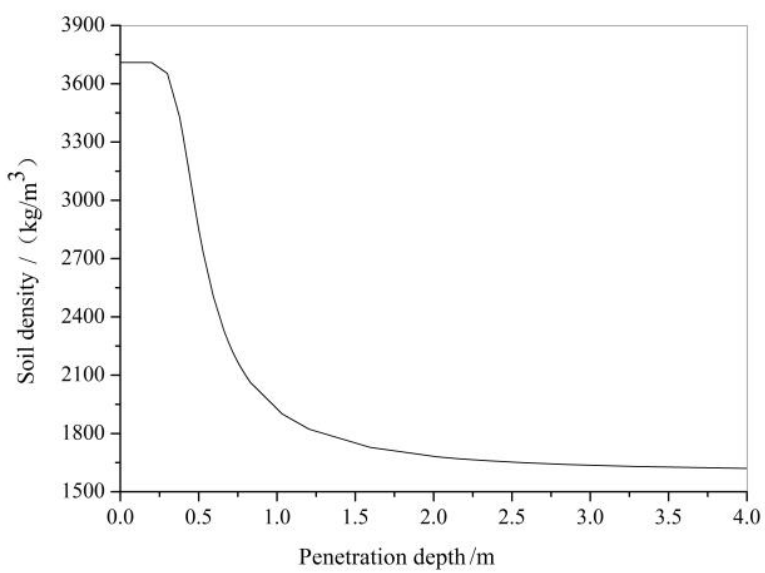

Figure 4. Variation law of soil density with penetration depth

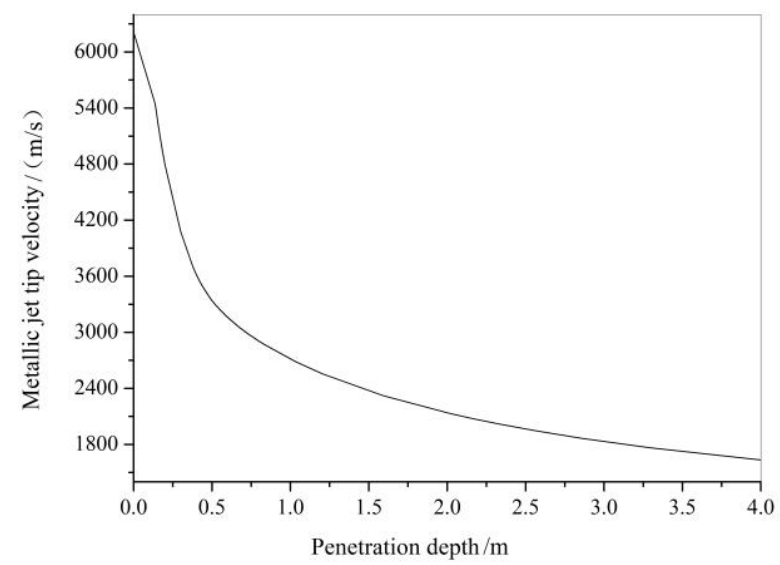

Figure 5. Variation law of jet tip velocity with penetration depth

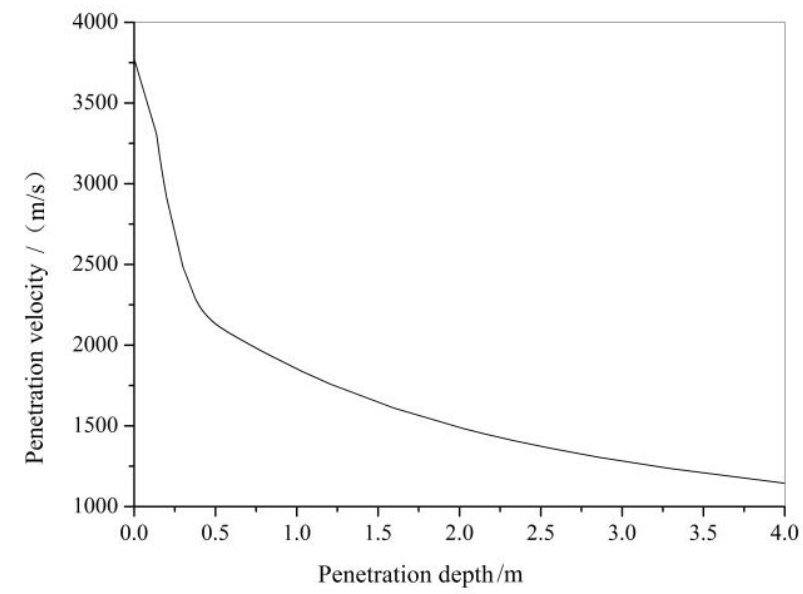

Figure 6. Variation law of penetration velocity with penetration depth 


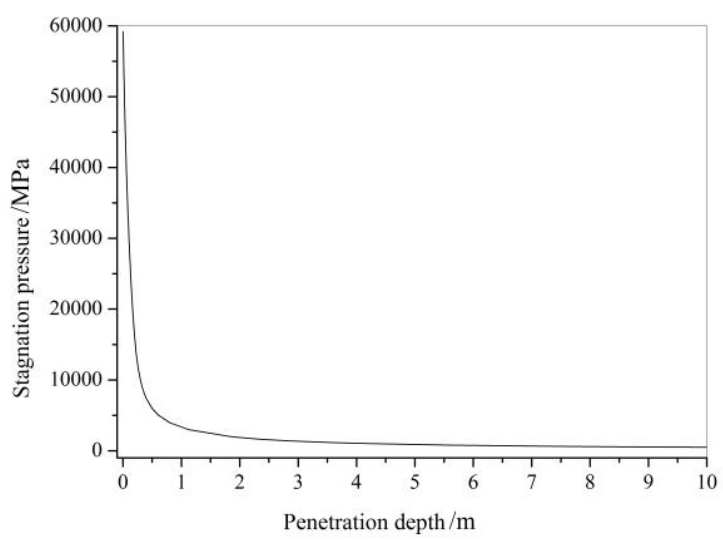

Figure 7. Variation law of stagnation pressure with penetration depth

As indicated in Fig.7,metallic jet might penetrate into soil as deep as $10 \mathrm{~m}$, which is attributed to ruling out soil strength, while after metallic jet velocity decreases to some extent, soil strength has become non-negligible. When stagnation pressure of metallic jet penetration into soil is smaller than the limit of soil strength, metallic jet stops penetration.

\section{Conclusion}

Based on theories of soil compression and metallic jet penetration, this paper built the dynamic model of metallic jet penetration into soil, and discussed application of the constructed model in study and analysis of metallic jet penetration into soil. The model constructed in the paper is capable of completely reflecting variation law of soil density, jet tip velocity, penetration velocity, penetration stagnation pressure along with penetration depth, indicating that in the research process of metallic jet penetration into soil, we could not simply regard the overall soil as a grid model, and must take a full consideration of soil compression properties.

\section{References}

[1] J.M.Li, Y.K.Ding and Y.Ke: Demolition Equipment and Materials, Vol. 37 (2008) No.3, p. 37.

[2] G.F.Song, C.G.Li and F.J.Xia: Initiating Explosive Device, (2009) No.6, p. 43.

[3] L.S.Dong, J.P.Yang and J.G.Gan: Science Technology and Engineering, Vol. 7 (2007) No.22, p. 5881.

[4] X.L.Fu and M.G.Shao: Transactions of the Chinese Society of Agricultural Engineering, Vol. 23 (2007) No.4, p. 1.

[5] Beijing Institute of Industries: Explosion and Its Effects (Volume II)(Beijing: National Defense Industry Press, 1979), p.84. 